\title{
GRÜSS TYPE INEQUALITIES INVOLVING THE GENERALIZED GAUSS HYPERGEOMETRIC FUNCTIONS
}

\author{
Abdullah Akkurt ${ }^{1}$, Seda Kilinç², Hüseyin Yildirim ${ }^{3}$ \\ $1,2,3$ Department of Mathematics \\ Faculty of Science and Arts \\ University of Kahramanmaraş \\ Sütçü İmam, 46000 Kahramanmaraş, TURKEY
}

\begin{abstract}
In this paper, we establish certain generalized Grüss type inequality for generalized fractional integral inequalities involving the generalized Gauss hypergeometric function. Moreover, we also consider their relevances for other related known results.
\end{abstract}

AMS Subject Classification: 26D10, 26A33

Key Words: integral inequalities, Grüss inequality, fractional integrals and fractional derivatives, Gauss hypergeometric functions

\section{Introduction and Preliminares}

In 1935, D. Grüss, proved the following integral inequality which gives an estimation of a product in terms of the product of integrals [6]:

$$
\begin{aligned}
& \left|\frac{1}{(b-a)} \int_{a}^{b} f(x) g(x) d x-\frac{1}{(b-a)} \int_{a}^{b} f(x) d x \frac{1}{(b-a)} \int_{a}^{b} g(x) d x\right| \\
& \quad \leq \frac{1}{4}(L-l)(M-m) ;
\end{aligned}
$$

provided that $f$ and $g$ are two functions which are defined and integrable on

Received: October 21, 2015

Published: March 9, 2016

$\S_{\text {Correspondence author }}$ (c) 2016 Academic Publications, Ltd.

url: www.acadpubl.eu 
$[a, b]$ and satisfying the condition

$$
l \leq f(x) \leq L, m \leq g(x) \leq M
$$

for all $x \in[a, b]$.

The costant $\frac{1}{4}$ is the best possible.

Definition 1. ([26,27]) Let $h(x)$ be an increasing and positive monotone function on $[0, \infty)$, also derivative $h(x)$ is continuous on $[0, \infty)$ and $h(0)=$ 0 . The space $X_{h}^{p}(0, \infty)(1 \leq p<\infty)$ of those real-valued Lebesque measurable functions $f$ on $[0, \infty)$ for which

$$
\|f\|_{X_{h}^{p}}=\left(\int_{0}^{\infty}|f(t)|^{p} h(t) d t\right)^{\frac{1}{p}}<\infty, \quad 1 \leq p \leq \infty
$$

and for the case $p=\infty$

$$
\|f\|_{X_{h}^{\infty}}=e s s \sup _{1 \leq t<\infty}\left[f(t) h^{\prime}(t)\right] .
$$

In particular, when $h(x)=x(1 \leq p<\infty)$ the space $X_{h}^{p}(0, \infty)$ coincides with the $L_{p}[0, \infty)$-space and also if we take $h(x)=\frac{x^{k+1}}{k+1}(1 \leq p<\infty, k \geq 0)$ the space $X_{h}^{p}(0, \infty)$ coincides with the $L_{p, k}[0, \infty)$-space.

Definition 2. ([28]) Let $(a, b)$ be a finite interval of the real line $\mathbb{R}$ and $\alpha>0$. Also let $h(x)$ be an increasing and positive monotone function on $(a, b]$, having a continuous derivative $h^{\prime}(x)$ on $(a, b)$. The left- and right-sided fractional integrals of a function $f$ with respect to another function $h$ on $[a, b]$ for which

$$
\left(J_{a^{+}, h}^{\alpha} f\right)(x):=\frac{1}{\Gamma(\alpha)} \int_{a}^{x}[h(x)-h(t)]^{\alpha-1} h^{\prime}(t) f(t) d t, x \geq a,
$$

and

$$
\left(J_{b^{-}, h}^{\alpha} f\right)(x):=\frac{1}{\Gamma(\alpha)} \int_{x}^{b}[h(t)-h(x)]^{\alpha-1} h^{\prime}(t) f(t) d t, x \leq b .
$$

Definition 3. Let $f \in X_{h}^{1}$. For $\alpha>0, \delta>-1, \beta, \eta \in \mathbb{R}$ and $h(x)$ be an increasing and positive monotone function on $(0, x]$, having a continuous 
derivative $h^{\prime}(x)$ on $(0, x)$. Then the generalized fractional integral $I_{h(t)}^{\alpha, \beta, \eta, \delta}$ of order $\alpha$ for real-valued continuous function $f(t)$, is defined by

$$
\begin{aligned}
I_{h(t)}^{\alpha, \beta, \eta, \delta}\{f(x)\} & =\frac{h(x)^{-\alpha-\beta-2 \delta}}{\Gamma(\alpha)} \int_{0}^{x} h(t)^{\delta}(h(x)-h(t))^{\alpha-1} \\
& \times\left({ }_{2} F_{1}\left(\alpha+\beta+\delta,-\eta ; \alpha ; 1-\frac{h(t)}{h(x)}\right)\right) h^{\prime}(t) f(t) d t,
\end{aligned}
$$

where the function ${ }_{2} F_{1}($.$) appearing as a kernel for the operator (1.7) is the$ Gaussian hypergeometric function defined by

$$
{ }_{2} F_{1}(a, b ; c ; h(t))=\sum_{n=0}^{\infty} \frac{(a)_{n}(b)_{n}}{(c)_{n}} \frac{[h(t)]^{n}}{n !},
$$

where,

$$
{ }_{2} F_{1}(0, b ; c ; h(t))={ }_{2} F_{1}(a, b ; c ; 0)=1 .
$$

The Pochhammer symbol $(a)_{n}$ is defined by $(n \in \mathbb{N})$,

$$
(a)_{n}=a(a+1) \cdots(a+n-1) ; \quad(a)_{0}=1 .
$$

Where $\mathbb{N}$ denotes the set of positive integers. The above integral (1.7) has the following commutative property:

$$
I_{h(t)}^{\alpha, \beta, \eta, \delta} I_{h(t)}^{a, b, c, d} f(x)=I_{h(t)}^{a, b, c, d} I_{h(t)}^{\alpha, \beta, \eta, \delta} f(x) .
$$

In the sequel, we use the following well-known result to establish our main results in the present paper:

$$
{ }_{2} F_{1}(a, b ; c ; 1)=\frac{\Gamma(c) \Gamma(c-a-b)}{\Gamma(c-a) \Gamma(c-b)} \quad\left(\Re(c-a-b)>0 ; c \in \Xi / \mathbb{Z}_{0}^{-}\right),
$$

where and $\Xi$ and $/ \mathbb{Z}_{0}^{-}$denotes the sets of complex numbers and nonpositive integers, respectively.

We define a fractional integral operator $K_{h(t)}^{\alpha, \beta, \eta, \delta}$ associated with the Gauss hypergeometric function as follows.

Definition 4. Let $f \in X_{h}^{1}$. For $\alpha>\max \{0,-(\delta+\eta+1)\}, \beta-1<\eta<$ $0, \beta<1$ and $\delta>-1$ we define a fractional integral $K_{h(t)}^{\alpha, \beta, \eta, \delta} f$ as follows:

$$
\left(K_{h(t)}^{\alpha, \beta, \eta, \delta} f\right)(x)=\frac{\Gamma(1-\beta) \Gamma(\alpha+\delta+\eta+1)}{\Gamma(\eta-\beta+1) \Gamma(\delta+1)} h(x)^{\beta+\delta}\left(I_{h(t)}^{\alpha, \beta, \eta, \delta} f\right)(x),
$$

where $I_{h(t)}^{\alpha, \beta, \eta, \delta}$ is the Gauss hypergeometric fractional integral of order $\alpha$ and is defined in the following. 
Definition 5. Two functions $f$ and $g$ are said to be synchronous functions on $[0, \infty)$ if

$$
A(x, y)=(f(x)-f(y))(g(x)-g(y)) \geq 0 ;
$$

for any $x, y \in[0, \infty)$.

Lemma 6. For $\mu>\max \{0,-(\eta-\beta)\}-1, \alpha>\max \{0,-(\delta+\eta+1)\}$; and $\eta-\beta>-1, \beta<1, \delta+\mu>-1, h(x)$ be an increasing and positive monotone function on $(0, x]$, having a continuous derivative $h(x)$ on $(0, x), h(0)=0$, we have

$$
\begin{aligned}
& K_{h(t)}^{\alpha, \beta, \eta, \delta}\left(h^{\mu}(x)\right) \\
& =\frac{\Gamma(1-\beta) \Gamma(\alpha+\delta+\eta+1) \Gamma(\delta+\mu+1) \Gamma(\mu-\beta+\eta+1)}{\Gamma(\eta-\beta+1) \Gamma(\delta+1) \Gamma(\mu-\beta+1) \Gamma(\mu+\delta+\alpha+\eta+1)} h^{\mu}(x)
\end{aligned}
$$

and

where $C$ is constant.

$$
K_{h(t)}^{\alpha, \beta, \eta, \delta}(C)=C
$$

Proof. Using the result (1.7), (1.12) reduces to

$$
\begin{aligned}
& K_{h(t)}^{\alpha, \beta, \eta, \delta}\left(h^{\mu}(x)\right)=\frac{\Gamma(1-\beta) \Gamma(\alpha+\delta+\eta+1)[h(x)]^{\beta+\delta}}{\Gamma(h(x)]^{-\alpha-\beta-2 \delta}} \frac{[(\alpha)}{\Gamma(\alpha)} \\
\times & \int_{0}^{x}[h(t)]^{\delta+\mu}(h(x)-h(t))^{\alpha-1}\left({ }_{2} F_{1}\left(\alpha+\beta+\delta,-\eta ; \alpha ; 1-\frac{h(t)}{h(x)}\right)\right) h^{\prime}(t) d t .
\end{aligned}
$$

Using (1.8), (1.16) reduces to the following form:

$$
\begin{aligned}
& K_{h(t)}^{\alpha, \beta, \eta}\left(h^{\mu}(x)\right) \\
& =\frac{\Gamma(1-\beta) \Gamma(\alpha+\delta+\eta+1)[h(x)]^{-\alpha-\delta}}{\Gamma(\eta-\beta+1) \Gamma(\delta+1) \Gamma(\alpha)} \\
& \times \sum_{n=0}^{\infty} \frac{(a+\beta+\delta)_{n}(-\eta)_{n}}{(\alpha)_{n} n !}[h(x)]^{-n} \int_{0}^{x}[h(t)]^{\delta+\mu}(h(x)-h(t))^{\alpha+n-1} h(t) d t \\
& =\frac{\Gamma(1-\beta) \Gamma(\alpha+\delta+\eta+1)[h(x)]^{-\alpha-\delta}}{\Gamma(\eta-\beta+1) \Gamma(\delta+1) \Gamma(\alpha)} \sum_{n=0}^{\infty} \frac{(a+\beta+\delta)_{n}(-\eta)_{n}}{(\alpha)_{n} n !}[h(x)]^{-n} \\
& \times \\
& =\frac{\Gamma(h(x)]^{\delta+\mu+\alpha+n+1-1} B(\delta+\mu+1, \alpha+n)}{\Gamma(\eta-\beta+1) \Gamma(\delta+1) \Gamma(\alpha+\delta+\mu+1)} \sum_{n=0}^{\infty} \frac{(a+\beta+\delta)_{n}(-\eta)_{n}}{(\alpha+\delta+\mu+1)_{n} n !} h^{\mu}(x) \\
& =\frac{\Gamma(1-\beta) \Gamma(\alpha+\delta+\eta+1) \Gamma(\delta+\mu+1)}{\Gamma(\eta-\beta+1) \Gamma(\delta+1) \Gamma(\alpha+\delta+\mu+1)}
\end{aligned}
$$




$$
\begin{aligned}
\times & {\left[{ }_{2} F_{1}(a+\beta+\delta,-\eta ; \alpha+\delta+\mu+1 ; 1)\right] h^{\mu}(x) } \\
= & \frac{\Gamma(1-\beta) \Gamma(\alpha+\delta+\eta+1) \Gamma(\delta+\mu+1)}{\Gamma(\eta-\beta+1) \Gamma(\delta+1) \Gamma(\alpha+\delta+\mu+1)} \\
\times & \frac{\Gamma(\alpha+\delta+\mu+1) \Gamma(\alpha+\delta+\mu+1-(a+\beta+\delta)-(-\eta))}{\Gamma(\alpha+\delta+\mu+1-(a+\beta+\delta)) \Gamma(\alpha+\delta+\mu+1-(-\eta))} h^{\mu}(x) \\
= & \frac{\Gamma(1-\beta) \Gamma(\alpha+\delta+\eta+1) \Gamma(\delta+\mu+1)}{\Gamma(\eta-\beta+1) \Gamma(\delta+1) \Gamma(\alpha+\delta+\mu+1)} \\
\times & \frac{\Gamma(\alpha+\delta+\mu+1) \Gamma(\mu+1-\beta+\eta)}{\Gamma(\mu+1-\beta) \Gamma(\alpha+\delta+\mu+1+\eta)} h^{\mu}(x) \\
= & \frac{\Gamma(1-\beta) \Gamma(\alpha+\delta+\eta+1) \Gamma(\delta+\mu+1) \Gamma(\mu-\beta+\eta+1)}{\Gamma(\eta-\beta+1) \Gamma(\delta+1) \Gamma(\mu-\beta+1) \Gamma(\mu+\delta+\alpha+\eta+1)} h^{\mu}(x)
\end{aligned}
$$

Proof is done.

To prove (1.15), we again use the result (1.7), and (1.12) reduces to

$$
\begin{aligned}
K_{h(t)}^{\alpha, \beta, \eta, \delta}(C) & =\frac{\Gamma(1-\beta) \Gamma(\alpha+\delta+\eta+1)[h(x)]^{\beta+\delta}}{\Gamma(\eta-\beta+1) \Gamma(\delta+1)} \\
& \times \frac{[h(x)]^{-\alpha-\beta-2 \delta}}{\Gamma(\alpha)} \int_{0}^{x}[h(t)]^{\delta}(h(x)-h(t))^{\alpha-1} \\
& \times\left({ }_{2} F_{1}\left(\alpha+\beta+\delta,-\eta ; \alpha ; 1-\frac{h(t)}{h(x)}\right)\right) h^{\prime}(t) C d t .
\end{aligned}
$$

Using (1.8), (1.18) gets the following form:

$$
\begin{aligned}
& K_{h(t)}^{\alpha, \beta, \eta, \delta}(C) \\
& =C \frac{\Gamma(1-\beta) \Gamma(\alpha+\delta+\eta+1)[h(x)]^{-\alpha-\delta}}{\Gamma(\eta-\beta+1) \Gamma(\delta+1)} \frac{1}{\Gamma(\alpha)} \\
& \times \sum_{n=0}^{\infty} \frac{(a+\beta+\delta)_{n}(-\eta)_{n}}{(\alpha)_{n} n !}[h(x)]^{-n} \int_{0}^{x}[h(t)]^{\delta}(h(x)-h(t))^{\alpha+n-1} h(t) d t \\
& =C \frac{\Gamma(1-\beta) \Gamma(\alpha+\delta+\eta+1)[h(x)]^{-\alpha-\delta}}{\Gamma(\eta-\beta+1) \Gamma(\delta+1)} \frac{1}{\Gamma(\alpha)} \sum_{n=0}^{\infty} \frac{(a+\beta+\delta)_{n}(-\eta)_{n}}{(\alpha)_{n} n !}[h(x)]^{-n} \\
& \times[h(x)]^{\delta+\alpha+n+1-1} B(\delta+1, \alpha+n) \\
& =C \frac{\Gamma(1-\beta) \Gamma(\alpha+\delta+\eta+1)}{\Gamma(\eta-\beta+1) \Gamma(\alpha+\delta+1)} \sum_{n=0}^{\infty} \frac{(a+\beta+\delta)_{n}(-\eta)_{n}}{(\alpha+\delta+1)_{n} n !} \\
& =C \frac{\Gamma(1-\beta) \Gamma(\alpha+\delta+\eta+1)}{\Gamma(\eta-\beta+1) \Gamma(\alpha+\delta+1)}\left[{ }_{2} F_{1}(\alpha+\beta+\delta,-\eta ; \alpha+\delta+1 ; 1)\right] \\
& =C \frac{\Gamma(1-\beta) \Gamma(\alpha+\delta+\eta+1)}{\Gamma(\eta-\beta+1) \Gamma(\alpha+\delta+1)} \frac{\Gamma(\alpha+\delta+1) \Gamma(1-\beta+\eta)}{\Gamma(1-\beta) \Gamma(\alpha+\delta+1+\eta)} \\
& =C
\end{aligned}
$$


This completes the proof of the Lemma 6.

Lemma 7. Let $g \in X_{h}^{1}$ and $m, M \in \mathbb{R}$ with $m \leq g(x) \leq M$. Then we have

$$
\begin{aligned}
& K_{h(t)}^{\alpha, \beta, \eta, \delta} g^{2}(x)-\left(K_{x}^{\alpha, \beta, \eta, \delta} \operatorname{tg}(x)\right)^{2} \\
& \quad=\left(M-K_{h(t)}^{\alpha, \beta, \eta, \delta} g(x)\right)\left(K_{h(t)}^{\alpha, \beta, \eta, \delta} g(x)-m\right)-K_{h(t)}^{\alpha, \beta, \eta, \delta}(M-g(x))(g(x)-m),
\end{aligned}
$$

for all $x \in[0, \infty) ; \alpha>0, \delta>-1$, and $\beta, \eta \in \mathbb{R}$ with $\alpha+\beta+\delta \geq 0$ and $\eta \leq 0$.

Proof. Let $g \in X_{h}^{1}$ and $m, M \in \mathbb{R} ; m \leq g(x) \leq M$, for all $x \in[0, \infty)$. Then, for any $u, v \in[0, \infty)$, we have

$$
\begin{gathered}
(M-g(u))(g(v)-m)+(M-g(v))(g(u)-m)-(M-g(u))(g(u)-m) \\
-(M-g(v))(g(v)-m)=g^{2}(u)+g^{2}(v)-2 g(u) g(v) .
\end{gathered}
$$

If $g \in X_{h}^{1}$, then $g$ is integrable on $[0, x], x>0$.

Thus multiplying (1.21) by

$$
\frac{[h(u)]^{\delta}(h(x)-h(u))^{\alpha-1}}{\Gamma(\alpha)} h^{\prime}(u)\left({ }_{2} F_{1}\left(\alpha+\delta+\beta,-\eta ; \alpha ; 1-\frac{h(u)}{h(x)}\right)\right)
$$

using $u \in(0, x) ; x>0$, and integrating with respect to $u$ from 0 to $x$, and then applying Definition 4 and Lemma 6, we obtain

$$
\begin{aligned}
& \left(M-K_{h(t)}^{\alpha, \beta, \eta, \delta} g(x)\right)(g(v)-m)+(M-g(v))\left(K_{h(t)}^{\alpha, \beta, \eta, \delta} g(x)-m\right) \\
& \quad-K_{h, \beta, \eta, \delta}^{\alpha, t)}(M-g(x))(g(x)-m)-(M-g(v))(g(v)-m) \\
& =K_{h(t)}^{\alpha, \beta, \eta, \delta} g^{2}(x)+g^{2}(v)-2 K_{h(t)}^{\alpha, \beta, \eta, \delta} g(x) g(v)
\end{aligned}
$$

Again multiplying (1.22) by

$$
\frac{[h(v)]^{\delta}(h(x)-h(v))^{\alpha-1}}{\Gamma(\alpha)} h(v)\left({ }_{2} F_{1}\left(\alpha+\delta+\beta,-\eta ; \alpha ; 1-\frac{h(v)}{h(x)}\right)\right),
$$

$v \in(0, x) ; x>0$, then integrating with respect to $v$ from 0 to $x$, we obtain the required result (1.20). This completes the proof of Lemma 7. 


\section{Main Results}

Theorem 8. Let $f$ and $g$ be two functions defined and integrable on $[a, b]$ with $f, g \in X_{h}^{1}$ and satisfying the condition (1.1) on $[0, \infty)$. Thus we have

$$
\left|K_{h(t)}^{\alpha, \beta, \eta, \delta} f g(x)-K_{h(t)}^{\alpha, \beta, \eta, \delta} f(x) K_{h(t)}^{\alpha, \beta, \eta, \delta} g(x)\right| \leq \frac{1}{4}(L-l)(M-m),
$$

for all $x \in[0, \infty) ; \alpha>0, \delta>-1$, and $\beta, \eta \in \mathbb{R}$ with $\alpha+\beta+\delta \geq 0$ and $\eta \leq 0$.

Proof. Let us define a function

$$
A(u, v)=(f(u)-f(v))(g(u)-g(v)) \quad(u, v \in[0, \infty)) .
$$

First multiplying (2.2) by

$$
\begin{aligned}
& \frac{(h(u) h(v))^{\delta}(h(x)-h(u))^{\alpha-1}(h(x)-h(v))^{\alpha-1}}{(\Gamma(\alpha))^{2}} h^{\prime}(u) h(v) \\
& \times\left({ } _ { 2 } F _ { 1 } ( \alpha + \delta + \beta , - \eta ; \alpha ; 1 - \frac { h ( u ) } { h ( x ) } ) \left({ }_{2} F_{1}\left(\alpha+\delta+\beta,-\eta ; \alpha ; 1-\frac{h(v)}{h(x)}\right)\right.\right.
\end{aligned}
$$

and then integrating twice with respect to $u$ and $v$ from 0 to $x$, we obtain the following result with the aid of (1.7), (1.12), and property (1.8):

$$
\begin{aligned}
& \frac{1}{(\Gamma(\alpha))^{2}} \int_{0}^{x} \int_{0}^{x}(h(u) h(v))^{\delta}(h(x)-h(u))^{\alpha-1}(h(x)-h(v))^{\alpha-1} \\
& \times\left({ }_{2} F_{1}\left(\alpha+\delta+\beta,-\eta ; \alpha ; 1-\frac{h(u)}{h(x)}\right)\right. \\
& \times\left({ }_{2} F_{1}\left(\alpha+\delta+\beta,-\eta ; \alpha ; 1-\frac{h(v)}{h(x)}\right) A(u, v) h^{\prime}(u) h^{\prime}(v) d u d v\right. \\
& =2 K_{h(t)}^{\alpha, \beta, \eta, \delta} f g(x)-2 K_{h(t)}^{\alpha, \beta, \eta, \delta} f(x) K_{h(t)}^{\alpha, \beta, \eta, \delta} g(x) .
\end{aligned}
$$

Making use of the well-known Cauchy-Schwarz inequality for a linear operator, we find that

$$
\begin{aligned}
& \left(K_{h(t)}^{\alpha, \beta, \eta, \delta} f g(x)-K_{h(t)}^{\alpha, \beta, \eta, \delta} f(x) K_{h(t)}^{\alpha, \beta, \eta, \delta} g(x)\right)^{2} \\
& \leq\left(K_{h(t)}^{\alpha, \beta, \eta, \delta} f^{2}(x)-\left(K_{h(t)}^{\alpha, \beta, \eta, \delta} f(x)\right)^{2}\right)\left(K_{h(t)}^{\alpha, \beta, \eta, \delta} g^{2}(x)-\left(K_{h(t)}^{\alpha, \beta, \eta, \delta} g(x)\right)^{2}\right) .
\end{aligned}
$$

Since

$$
(L-f(x))(f(x)-l) \geq 0 \quad \text { and } \quad(M-f(x))(f(x)-m) \geq 0,
$$


we therefore have

$$
K_{h(t)}^{\alpha, \beta, \eta, \delta}(L-f(x))(f(x)-l) \geq 0 \quad \text { and } \quad K_{h(t)}^{\alpha, \beta, \eta, \delta}(M-f(x))(f(x)-m) \geq 0 .
$$

Thus by using Lemma 7, we have

$$
K_{h(t)}^{\alpha, \beta, \eta, \delta} f^{2}(x)-\left(K_{h(t)}^{\alpha, \beta, \eta, \delta} f(x)\right)^{2} \leq\left(L-K_{h(t)}^{\alpha, \beta, \eta, \delta} f(x)\right)\left(K_{h(t)}^{\alpha, \beta, \eta, \delta} f(x)-l\right)
$$

and

$$
K_{h(t)}^{\alpha, \beta, \eta, \delta} g^{2}(x)-\left(K_{h(t)}^{\alpha, \beta, \eta, \delta} g(x)\right)^{2} \leq\left(M-K_{h(t)}^{\alpha, \beta, \eta, \delta} g(x)\right)\left(K_{h(t)}^{\alpha, \beta, \eta, \delta} g(x)-m\right) .
$$

Using the inequalities (2.6) and (2.7), (2.4) reduces to the following form:

$$
\begin{aligned}
& \left(K_{h(t)}^{\alpha, \beta, \eta, \delta} f g(x)-K_{h(t)}^{\alpha, \beta, \eta, \delta} f(x) K_{h(t)}^{\alpha, \beta, \eta, \delta} g(x)\right)^{2} \\
& \leq\left(L-K_{h, \beta, \eta, \delta}^{\alpha, \beta} f(x)\right)\left(K_{h(t)}^{\alpha, \beta, \eta, \delta} f(x)-l\right)\left(M-K_{h(t)}^{\alpha, \beta, \eta, \delta} g(x)\right) \\
& \quad \times\left(K_{h(t)}^{\alpha, \beta, \eta, \delta} g(x)-m\right) .
\end{aligned}
$$

Applying the well-known inequality $4 a b \leq(a+b)^{2}$; and using $a, b \in \mathbb{R}$ in the right-hand side of the inequality (2.8),

$$
\begin{aligned}
& \left(K_{h(t)}^{\alpha, \beta, \eta, \delta} f g(x)-K_{h(t)}^{\alpha, \beta, \eta, \delta} f(x) K_{h(t)}^{\alpha, \beta, \eta, \delta} g(x)\right)^{2} \\
& \leq \frac{\left[\left(L-K_{h(t)}^{\alpha, \beta, \eta, \delta} f(x)+K_{h(t)}^{\alpha, \beta, \eta, \delta} f(x)-l\right]^{2}\right.}{4} \frac{\left[M-K_{h(t)}^{\alpha, \beta, \eta, \delta} g(x)+K_{h(t)}^{\alpha, \beta, \eta, \delta} g(x)-m\right]^{2}}{4}
\end{aligned}
$$

and simplifying it,

$$
\begin{aligned}
& \left(K_{h(t)}^{\alpha, \beta, \eta, \delta} f g(x)-K_{h(t)}^{\alpha, \beta, \eta, \delta} f(x) K_{h(t)}^{\alpha, \beta, \eta, \delta} g(x)\right) \\
& \leq \frac{1}{4}(L-l)(M-m)
\end{aligned}
$$

we obtain the required result (2.1). This completes the proof of Theorem 8 .

Theorem 9. Let $f$ and $g$ be two synchronous functions on $[0, \infty)$. Then the following inequality holds:

$$
K_{h(t)}^{\alpha, \beta, \eta, \delta} f g(x) \geq K_{h(t)}^{\alpha, \beta, \eta, \delta} f(x) K_{h(t)}^{\alpha, \beta, \eta, \delta} g(x)
$$

for all $x \in[0, \infty) ; \alpha>0, \delta>-1$, and $\beta, \eta \in R$ with $\alpha+\beta+\delta \geq 0$ and $\eta \leq 0$. 
Proof. For the synchronous function $f$ and $g$, the inequality (1.13) holds for all $u, v \in[0, \infty)$. This implies that

$$
f(u) g(u)-f(v) g(v) \geq f(u) g(v)+f(v) g(u) .
$$

Following the procedure of the Lemma 7 for applying the fractional integral $K_{h(t)}^{\alpha, \beta, \eta, \delta}$, after a little simplification, we arrive at the required result (2.9). This completes the proof of Theorem 9 .

\section{Concluding Remarks}

We consider some consequences of the results derived in the previous section.

$$
\begin{aligned}
I_{0, h(t)}^{\alpha, \beta, \eta}\{f(t)\} & =I_{h(t)}^{\alpha, \beta, \eta, 0}\{f(t)\} \\
& =\frac{[h(x)]^{-\alpha-\beta}}{\Gamma(\alpha)} \int_{0}^{t}(h(t)-h(\tau))^{\alpha-1} \\
& \times\left({ }_{2} F_{1}\left(\alpha+\beta,-\eta ; \alpha ; 1-\frac{h(\tau)}{h(t)}\right)\right) h^{\prime}(\tau) f(\tau) d \tau
\end{aligned}
$$

for $\delta=0$,

$$
\begin{aligned}
I^{\alpha, \eta}\{f(t)\} & =I_{h(t)}^{\alpha, 0, \eta, 0}\{f(t)\} \\
& =\frac{[h(x)]^{-\alpha-\eta}}{\Gamma(\alpha)} \int_{0}^{t}(h(t)-h(\tau))^{\alpha-1}[h(\tau)]^{\eta} h^{\prime}(\tau) f(\tau) d \tau,
\end{aligned}
$$

for $\delta=0$ and $\beta=0$, and

$$
\begin{aligned}
R^{\alpha}\{f(t)\} & =I_{h(t)}^{\alpha,-\alpha, \eta, 0}\{f(t)\} \\
& =\frac{1}{\Gamma(\alpha)} \int_{0}^{t}(h(t)-h(\tau))^{\alpha-1} h^{\prime}(\tau) f(\tau) d \tau \quad(\alpha>0)
\end{aligned}
$$

for $\delta=0$ and $\beta=-\alpha$.

Here if we choose $h(x)=x$, following we, the operator (1.7) would reduce immediately to the extensively investigated Saigo, Erdélyi-Kober, and Riemann-Liouville type fractional integral operators, respectively, given by the 
following relationships (see also [17], [19], [20]):

$$
\begin{aligned}
I_{0, t}^{\alpha, \beta, \eta}\{f(t)\} & =I_{t}^{\alpha, \beta, \eta, 0}\{f(t)\} \\
& =\frac{x^{-\alpha-\beta}}{\Gamma(\alpha)} \int_{0}^{t}(t-\tau)^{\alpha-1} \\
& \times\left({ }_{2} F_{1}\left(\alpha+\beta,-\eta ; \alpha ; 1-\frac{\tau}{t}\right)\right) f(\tau) d \tau
\end{aligned}
$$

for $\delta=0$,

$$
\begin{aligned}
I^{\alpha, \eta}\{f(t)\} & =I_{t}^{\alpha, 0, \eta, 0}\{f(t)\} \\
& =\frac{x^{-\alpha-\eta}}{\Gamma(\alpha)} \int_{0}^{t}(t-\tau)^{\alpha-1} \tau^{\eta} f(\tau) d \tau,
\end{aligned}
$$

for $\delta=0$ and $\beta=0$, and

$$
\begin{aligned}
R^{\alpha}\{f(t)\} & =I_{t}^{\alpha,-\alpha, \eta, 0}\{f(t)\} \\
& =\frac{1}{\Gamma(\alpha)} \int_{0}^{t}(t-\tau)^{\alpha-1} f(\tau) d \tau \quad(\alpha>0),
\end{aligned}
$$

for $\delta=0$ and $\beta=-\alpha$.

Remark 10. We obtain the special cases of the operator $K_{h(t)}^{\alpha, \beta, \eta, \delta}$ as follows by setting $\delta=0, \delta=\beta=0, \delta=0, \beta=-\alpha$ and $h(x)=x$ in Definition 3. Immediately Definition 3. would reduce to the Saigo, ErdélyiKober, and Riemann-Liouville type fractional integral operators, respectively, given as follows:

$$
\begin{gathered}
\left(K_{t}^{\alpha, \beta, \eta} f\right)(x)=\frac{\Gamma(1-\beta) \Gamma(\alpha+\eta+1)}{\Gamma(\eta-\beta+1)} x^{\beta}\left(I_{0, t}^{\alpha, \beta, \eta} f\right)(x), \\
\left(K_{t}^{\alpha, \eta} f\right)(x)=\frac{\Gamma(\alpha+\eta+1)}{\Gamma(\eta+1)} x^{\beta}\left(I^{\alpha, \eta} f\right)(x)
\end{gathered}
$$

and

$$
\left(K_{t}^{\alpha} f\right)(x)=\frac{\Gamma(\alpha+1)}{x^{\alpha}}\left(R^{\alpha} f\right)(x)
$$

where $\left(I_{0, t}^{\alpha, \beta, \eta}\right),\left(I^{\alpha, \eta}\right)$ and $\left(R^{\alpha}\right)$ are given by (3.1), (3.2), and (3.3), respectively.

We conclude our present investigation by remarking further that the results obtained here are useful in deriving various fractional integral inequalities involving such relatively more familiar fractional integral operators. For example, 
if we consider $\delta=0, h(x)=x$ and make use of (3.1), Theorems 8. and 9. provide, respectively, the known fractional integral inequalities due to Kalla and Rao [12].

Similarly, if we choose $\delta=0, \beta=0$ and $h(x)=x$ in Theorems 8 . and 9 and making use of the relation (3.2), Theorems 8. and 9. provide, respectively, the known fractional integral inequalities owing to Kalla and Rao [12].

Finally, if we choose $\delta=0, h(x)=x$ and $\beta=-\alpha$ in Theorems 8. and 9. yields the known result owing to Dahmani et al. [5].

\section{References}

[1] S. Belarbi, and Z. Dahmani, On some new fractional integral inequalities, J. Inequal. Pure Appl. Math. 10(3), Article 86 (2009)

[2] G.A. Anastassiou, Advances on Fractional Inequalities, Springer Briefs in Mathematics. Springer, New York (2011)

[3] Z. Dahmani, O. Mechouar, S. Brahami, Certain inequalities related to the Chebyshev's functional involving a type Riemann-Liouville operator, Bull. Math. Anal. Appl. 3(4), 38-44 (2011)

[4] Z. Dahmani, New inequalities in fractional integrals, Int. J. Nonlinear Sci. 9, 493-497 (2010)

[5] Z. Dahmani, L. Tabharit, S. Taf, New generalisations of Grüss inequality using RiemannLiouville fractional integrals, Bull. Math. Anal. Appl. 2(3), 93-99 (2010)

[6] D. Grüss, Über das maximum des absoluten Betrages von $\frac{1}{b-a} \int_{a}^{b} f(x) g(x) d x-$ $\frac{1}{(b-a)^{2}} \int_{a}^{b} f(x) d x \int_{a}^{b} g(x) d x$, Math. Z. 39, 215-226 (1935)

[7] S.S. Dragomir, Some integral inequalities of Grüss type, Indian J. Pure Appl. Math. 31(4), 397-415 (2002)

[8] J.C. Kuang, Applied Inequalities, Shandong Sciences and Technology Press, Jinan (2004)

[9] D.S Mitrinović, Analytic Inequalities, Springer, Berlin (1970)

[10] W.T. Sulaiman, Some new fractional integral inequalities, J. Math. Anal. 2(2), 23-28 (2011)

[11] D. Baleanu, S.D. Purohit and P. Agarwal, On fractional integral inequalities involving hypergeometric operators, Chin. J. Math. 2014, Article ID 609476 (2014). doi: $10.1155 / 2014 / 609476$

[12] S.L. Kalla and A. Rao, On Grüss type inequalities for a hypergeometric fractional integral, Matematiche LXVI(1), 57-64 (2011)

[13] D.S. Mitrinović, J.E. Pečarić and A.M. Fink, Classical and New Inequalities in Analysis, Kluwer Academic, Dordrecht (1993)

[14] A.McD. Mercer, An improvement of the Grüss inequality, J. Inequal. Pure Appl. Math. 6(4), 1-4 (2005) 
[15] B.G. Pachpatte, On Grüss type integral inequalities, J. Inequal. Pure Appl. Math. 3(1), 1-5 (2002)

[16] B.G. Pachpatte, A note on Chebyshev-Grüss inequalities for differential equations, Tamsui Oxford Univ. J. Math. Sci. 22(1), 29-36 (2006)

[17] L. Curiel and L. Galué, A generalization of the integral operators involving the Gauss' hypergeometric function, Rev. Téc. Fac. Ing., Univ. Zulia 19(1), 17-22 (1996)

[18] I.H. Dimovski, Operational calculus for a class of differential operators. C. R. Acad. Bulgare Sci. 19, 1111-1114 (1966)

[19] V.S. Kiryakova, Generalized Fractional Calculus and Applications. Pitman Res. Notes Math. Ser., vol. 301. Longman, Harlow (1994)

[20] M. Saigo, A remark on integral operators involving the Gauss hypergeometric functions. Math. Rep. Kyushu Univ. 11, 135-143 (1978)

[21] U.N. Katugampola, New Approach to a Generalized Fractional Fntegral, Appl. Math. Comput. 218(3), (2011), 860-865.

[22] S.G. Samko, A.A. Kilbas and O.I. Marichev, Fractional Integrals and Derivatives, Theory and Applications, Gordon and Breach, Yverdon et alibi, 1993.

[23] A. Akkurt and H. Yıldırım, Genelleştirilmiş Fractional İntegraller İçin Feng Qi Tipli İntegral Eşitsizlikleri Üzerine, IAAOJ, Scientific Science, 2013,1(2), 22-25

[24] H. Yıldırım and Z. Kırtay, Ostrowski Inequality for Generalized Fractional Integral and Related Inequalities, Malaya Journal of Matematik, 2014.

[25] A. Akkurt, Z. Kaçar and H. Yıldırım, Generalized Fractional Integral Inequalities for Continuous Random Variables, Journal of Probability and Statistics, 2015.

[26] E. Kacar, H. Yıldırım, Grüss Type Integral Inequalities for Generalized RiemannLiouville Fractional Integrals, IJPAM., vol.101 no.1 (2015) pp. 55-70.

[27] S. Kılınç, H. Yıldırım, Generalized Fractional Integral Inequalities Involving Hipergeometric Operators, IJPAM., vol.101 no.1 (2015) pp.71-82.

[28] A.A. Kilbas, H.M. Srivastava, J.J. Trujillo, Theory and Applications of Fractional Differantial Equations, Elsevier B.V., Amsterdam, 2006. 